\title{
Immunohistochemical Identification of Muscle Fiber Types in Mice Tibialis Anterior Sections
}

\author{
Vanitha V. Rao ${ }^{1, \$, *}$ and Abhishek Mohanty ${ }^{2, *}$
}

${ }^{1}$ Department of Biochemistry, National University of Singapore, Kent Ridge, Singapore; ${ }^{2}$ Department of Molecular Oncology, MVR Cancer Center and Research Institute, Kozhikode, Kerala, India;'Pandorum Technologies Pvt. Ltd., Centre for Cellular and Molecular Platforms, NCBS-TIFR Campus, GKVK Post, Bellary Road, Bangalore 560065, India

*For correspondence: venustas06@gmail.com; abhishek.m.iisc@gmail.com

[Abstract] Mammalian skeletal muscle is a metabolically active tissue that is made up of different types of muscle fibers. These myofibers are made up of important contractile proteins that provide force during contraction of the muscle like actin and myosin. Murine myofibers have been classified into 4 types: Type I, Type Ila, Type IIb and Type IIX. Each muscle fiber has been identified with specific type of MyHC expressed, which in turn gives differential contractility to the muscle.

There have been well-known methodologies to identify different myofibers: histochemical myosin ATPase staining which uses the differential ATPase activity between slow and fast fibers, quantification of metabolic enzymes like malate dehydrogenase and lactate dehydrogenase on specific fragments of muscle fibers. The drawback of these techniques is that they cannot differentiate the subtypes of myofibers, for example, Type Ila and Type Ilb. They should be used in conjunction with other known histochemical staining techniques. Here, we devise a direct and robust immunohistochemical staining methodology that utilizes the differential expression of $\mathrm{MyHC}$ isoforms in different myofibers types, thus efficiently distinguishing the heterogeneity of the muscle fibers. We use antibodies that specifically recognize Type I, Type Ila and Type Ilb fibers on serially cut frozen mouse tibialis anterior sections that can be quantified by Image J software.

Keywords: Muscle fibers, Fiber-typing, Immunohistochemistry, Tibialis anterior muscle, Myosin heavy chain isoforms, murine myofiber heterogeneity

[Background] Skeletal muscle is a highly organized tissue composed of several distinct compartments of muscle fibers. Each muscle fiber is a multinucleated single cell, enclosed by sarcolemma, and is predominantly made up of myofibrils (Seeley et al., 2007). Muscle fibers that have low contraction speed are called slow twitch or type I fibers. Slow twitch fibers have high mitochondrial density and express myosin isoform Myh7 (Lompre et al., 1984; Talmadge and Roy, 1993). Type II fibers are divided into type Ila and type Ilb fibers. Both of these fibers have high contraction speed, thus generating greater force compared to type I fibers. Type lla fibers have moderately high contraction speed and are anaerobic in nature. Type lla fibers also have high density of mitochondria with increased oxidative potential. Type lla fibers predominantly contain myosin isoform Myh2 in mammals. Type Ilb fibers have very high contraction speed and are anaerobic in nature. Type Ilb fibers have low mitochondrial density and predominantly contain myosin isoform Myh4. Muscle fibers that are neither 
Type Ila nor Type Ilb fibers are called Type Ilx or Type Ild, and have high expression in the diaphragm and hence is omitted from this study (Schiaffino and Reggiani, 1994; Westerblad et al., 2010). Mouse tibialis anterior, a hind limb skeletal muscle made of Type I, Type Ila and Type Ilb myofibers serves as a suitable muscle to study the robustness of the immunohistochemical staining procedure.

Other methodologies to distinguish the different muscle fiber types are myosin ATPase histochemical staining of frozen muscle sections pre-incubated in alkaline $\mathrm{pH}$ showing light staining for Type I fibers and dark staining for Type II fibers. Muscle sections pre-incubated in acidic pH showed dark ATPase staining for Type I fibers, light staining for Type Ila muscle fibers and intermediate staining for Type Ilb fibers (Brooke and Kaiser, 1970, Guth and Samaha 1970). Quantitative estimation of mitochondrial enzymes like glycerophosphate oxidase (GP-OX) and succinate dehydrogenase (SDH) have been used to distinguish Type Ila and Type Ilb fibers in mouse and rabbit tibialis anterior muscle but gave variable ratios of enzyme activities in mice and rabbit muscles (Reichmann and Pette, 1984). There have been quantification methodologies of specific metabolic enzymes like lactate dehydrogenase and malate dehydrogenase on frozen serial sections of muscles (Hintz et al., 1984). However, these protocols are extensive and may give non-specific signals. The protocol detailed here uses specific antibodies that recognize different Myosin Heavy chain isoforms on serially cut frozen tibialis anterior mid-belly region sections that is readily detected with a suitable secondary antibody. After image acquisition, the number of each fiber type is quantified using ImageJ software.

\section{Materials and Reagents}

1. High Profile microtome blades 818 (Leica Biosystems, Germany, catalog number: 14035838926)

2. Microscope glass slides (Globe Scientific, USA, catalog number: GS-1308)

3. Camel hair brushes

4. Syringe plunger

5. $1.5 \mathrm{ml}$ microfuge tubes (USA Scientific, catalog number: 1615-5500)

6. Liquid blocker Pap pen (Sigma Aldrich, catalog number: Z377821)

7. Freshly dissected mouse hind limb tibialis anterior muscle

8. Myh fast $2 \mathrm{~A}$ antibody (Developmental Studies Hybridoma Bank, catalog number: 2F7)

9. Myh fast 2B antibody (Developmental Studies Hybridoma Bank, lowa City, IA, catalog number: 10F5)

10. Myh slow (type1) antibody (Developmental Studies Hybridoma Bank, lowa City, IA, catalog number: A4.840)

11. Biotinylated sheep anti-mouse IgG antibody (Abcam, catalog number: ab6807)

12. Tissue-Tek Optimal cutting temperature (OCT) compound (Sakura Finetek, USA, catalog number: 4583)

13. Isopentane (Alfa Aesar, catalog number: 19387, CAS number: 78-78-4)

14. Liquid Nitrogen 
15. Bovine serum albumin (BSA) (Roche, Switzerland, catalog number: 10711454001)

16. Normal sheep serum (NSS) (Sigma-Aldrich, catalog number: S22)

17. Tween 20 (RPI Corp., USA, catalog number: P20370, CAS number: 9005-64-5)

18. 4',6-diamidino-2-phenylindole Dihydrochloride (DAPI) (Invitrogen, catalog number: D1306)

19. ProLong Gold Anti-fade solution (Invitrogen, catalog number: P10144)

20. Streptavidin Alexa Fluor 488 conjugate (Invitrogen, catalog number: S-11223)

21. Sodium phosphate, monobasic

22. Sodium phosphate, dibasic

23. $37 \%$ Formaldehyde (Sigma-Aldrich, catalog number: 252549 , CAS number: $50-00-0$ )

24. Milli-Q water

25. Blocking mixture (see Recipes)

26. Diluent (see Recipes)

27. $10 \%$ Buffered formalin (see Recipes)

28. $0.2 \mu \mathrm{g} / \mathrm{ml}$ DAPI in 1x PBS (see Recipes)

29. 1x Phosphate buffered saline (PBS), pH 7.4 (see Recipes)

\section{Equipment}

1. Rotary Cryostat Microtome (CM1950, Leica Biosystems, Germany)

2. Orbital shaker (Boeco, Germany, Ref: BOE 8069200)

3. Leica CTR 6500 microscope equipped with Leica DFC 310 FX camera

4. Cryostat

\section{Software}

1. Image J (Fiji, https://imagej.nih.gov/ij/)

\section{Procedure}

A. Sample preparation

1. Dissect hind limb tibialis anterior muscle and remove the surrounding connective tissue.

2. Holding the tendon side of the muscle, place the tissue on the flat side of the syringe plunger.

3. Cover the tissue with OCT compound and immediately snap-freeze the tissue by immersing in isopentane cooled with liquid nitrogen for $30-40 \mathrm{~s}$.

4. Store the frozen tissues at $-80^{\circ} \mathrm{C}$ for future use.

5. Cut $10 \mu \mathrm{m}$ transverse serial sections at the mid-belly region of the muscle in a cryostat maintained at $-20^{\circ} \mathrm{C}$.

6. Support the sections while cryosectioning with a brush and pick the sections by placing a glass slide (kept at room temperature) in very close proximity to the sections. 
B. Immunostaining of the muscle sections

1. Draw a perimeter around the tissue section with a liquid blocker pen. This helps in staining multiple tissue sections on a glass slide and also reduces the volume of diluent required.

2. Add freshly prepared blocking solution (minimum of $200 \mu \mathrm{l} / \mathrm{section}$ ) onto sections to completely cover it and incubate at room temperature for an hour on low speed orbital shaker.

3. Prepare 1:25 dilutions of Myh fast 2A, Myh fast 2B and Myh slow (type I) primary antibody in separate $1.5 \mathrm{ml}$ microcentrifuge tubes with the diluent and incubate the sections overnight at $4{ }^{\circ} \mathrm{C}$ on a low speed orbital shaker.

4. Wash the sections with $1 \mathrm{x}$ PBS 3 times for 5 min each to remove the non-specific antibody bound to the sections.

5. Fix the primary antibody staining with $10 \%$ buffered formalin for $5 \mathrm{~min}$ and then wash three times with 1x PBS for five min each. It is recommended (specifically for DSHB MyHC primary antibodies) that the fixation should not be performed before primary antibody incubation, as this would disrupt the efficient binding of primary antibodies to different $\mathrm{MyHC}$ in the frozen sections. Hence fixation is performed after primary antibody incubation step.

6. Incubate the sections with 1:200 dilution of biotinylated sheep anti-mouse IgG secondary antibody made in diluent for one hour at room temperature with shaking.

7. Wash the sections with $1 \times$ PBS 3 times for 5 min each and incubate with 1:400 dilution of streptavidin Alexa Fluor 488 conjugate tertiary antibody in $0.2 \%$ BSA and 0.1\% PBS-Tween 20 ( $0.1 \%$ Tween 20 made in $1 \times$ PBS) for one hour at room temperature in the dark.

8. Wash the sections with $1 \times$ PBS twice for 5 min each.

9. Stain the myonuclei with DAPI made in $1 \mathrm{x}$ PBS diluted at 1:5,000 for 5 min and rinse thoroughly with $1 \times$ PBS.

10. Air-dry the slides completely to mount with ProLong Gold Anti-fade mountant solution and allow it to dry overnight in the dark.

11. Visualize using Leica CTR 6500 fluorescent microscope, equipped with the Leica DFC 310 FX camera under the FITC channel with wavelengths of excitation at $488 \mathrm{~nm}$ and emission at 536 nm.

\section{Data analysis}

To quantify the number of positive myofibers of each antibody used (Myh fast 2A, Myh fast 2B and Myh slow (type I), the images are imported into ImageJ (Fiji) and brightly stained muscle fibers from each antibody stained sections are counted. An example of this can be found in Figure 7 of the original research article Rao et al., 2019, which is again mentioned in this manuscript as Figure 1. The values were expressed as mean muscle fiber number \pm SE of two animals. Level of significance was calculated using two-tailed Student's $t$-test in comparison to wild-type muscle $\left.{ }^{* * *} P<0.001\right)$ (Rao et al., 2019). 


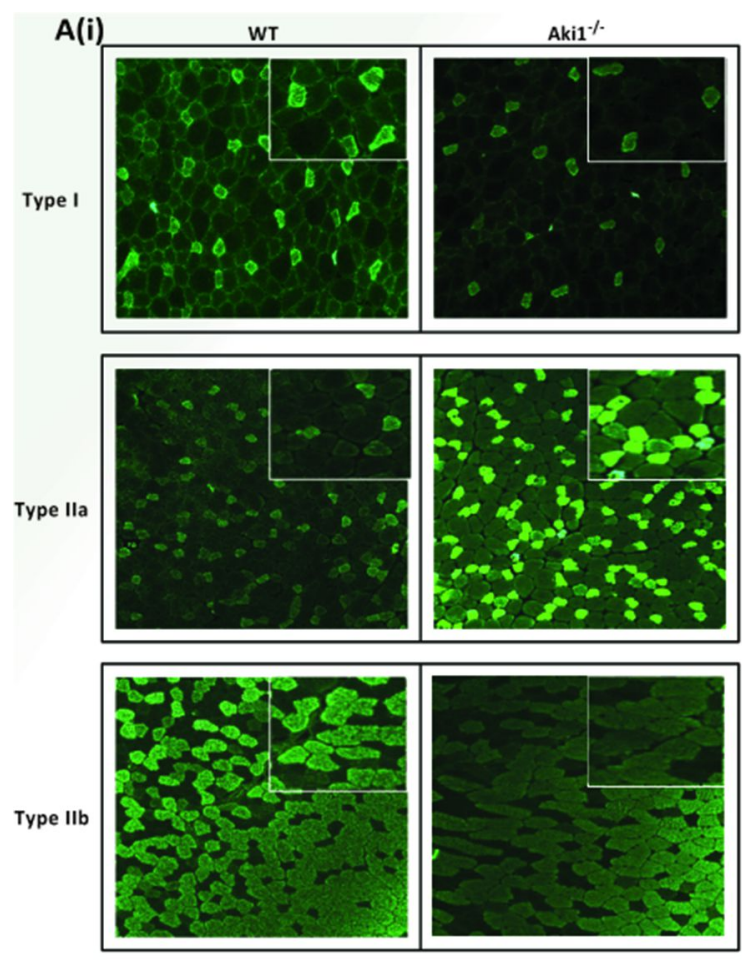

Figure 1. Showing the different types of MyHC (type I, type lla, and type Ilb) staining on Akirin1 knockout (Aki1 ${ }^{-1}$ ) and wild-type (WT) tibialis anterior muscle sections (Adapted from Rao et al., 2019).

\section{Recipes}

1. Blocking mixture

$0.5 \%$ Triton X-100

$0.2 \% \mathrm{BSA}$

$10 \%$ NSS

1x PBS

2. Diluent

\section{$0.2 \% \mathrm{BSA}$}

\section{$5 \%$ NSS}

$0.1 \%$ Tween 20 (made in $1 \times$ PBS)

3. $10 \%$ Buffered formalin

Sodium phosphate, monobasic

Sodium phosphate, dibasic

$37 \%$ Formaldehyde

Milli-Q water

4. $0.2 \mu \mathrm{g} / \mathrm{ml}$ DAPI in $1 \times$ PBS

a. Make stock concentration of $1 \mathrm{mg} / \mathrm{ml}$ DAPI

b. Take $2 \mu \mathrm{l}$ of the stock and make up to $10 \mathrm{ml}$ with $1 \times$ PBS 
5. 1x Phosphate buffered saline (PBS), $\mathrm{pH} 7.4$

Distilled water

Sodium Chloride

Potassium Chloride

Sodium phosphate, dibasic

Potassium dihydrogen phosphate

Adjust the $\mathrm{pH}$ to 7.4 with $\mathrm{HCl}$

Make up the volume to $1 \mathrm{~L}$
$800 \mathrm{ml}$

$8 \mathrm{~g}$

$0.2 \mathrm{~g}$

$1.44 \mathrm{~g}$

$0.24 \mathrm{~g}$

\section{Acknowledgments}

This work was supported by the funding agency: BMRC 07/1/21/19/521, NMRC/EDG/0026/2008, and Startup grant. The authors acknowledge National University of Singapore for the scholarship provided to Vanitha V. Rao throughout the course of her $\mathrm{PhD}$. The authors acknowledge the original research article Rao et al., 2019 from which this protocol was first discussed.

\section{Competing interests}

The authors declare no financial and non-financial competing interests.

\section{Ethics}

The animals used in the original research article were housed at the Nanyang Technological University Animal House, Singapore. All experiments were performed as per the approved protocols of Institutional Animal Ethics Committee (Singapore) with approval number ARFSBS/NIE-A0270.

\section{References}

1. Brooke, M. H. and Kaiser, K. K. (1970). Three "myosin adenosine triphosphatase" systems: the nature of their pH lability and sulfhydryl dependence. J Histochem Cytochem 18(9): 670-672.

2. Guth, L. and Samaha, F. J. (1970). Procedure for the histochemical demonstration of actomyosin ATPase. Exp Neurol 28(2): 365-367.

3. Hintz, C.S., Coyle, E.F., Kaiser, K.K., Chi, M.M., Lowry, O.H. (1984). Comparison of muscle fiber typing byquantitative enzyme assays and by myosin ATPase staining. $J$ Histochem Cytochem 32(6):655-660.

4. Lompre, A. M., Nadal-Ginard, B. and Mahdavi, V. (1984). Expression of the cardiac ventricular alpha- and beta-myosin heavy chain genes is developmentally and hormonally regulated. $J$ Biol Chem 259(10): 6437-6446. 
5. Rao, V. V., Sangiah, U., Mary, K. A., Akira, S. and Mohanty, A. (2019). Role of Akirin1 in the regulation of skeletal muscle fiber-type switch. J Cell Biochem. doi: 10.1002/jcb.28406.

6. Reichmann, H. and Pette, D. (1984). Glycerolphosphate oxidase and succinate dehydrogenase activities in IIA and IIB fibres of mouse and rabbit tibialis anterior muscles. Histochemistry 80(5): 429-433.

7. Schiaffino, S. and Reggiani, C. (1994). Myosin isoforms in mammalian skeletal muscle. J Appl Physiol (1985) 77(2): 493-501.

8. Seeley, M., Huang, W., Chen, Z., Wolff, W. O., Lin, X. and Xu, X. (2007). Depletion of zebrafish titin reduces cardiac contractility by disrupting the assembly of Z-discs and A-bands. Circ Res 100(2): 238-245.

9. Talmadge, R. J. and Roy, R. R. (1993). Electrophoretic separation of rat skeletal muscle myosin heavy-chain isoforms. J Appl Physiol (1985) 75(5): 2337-2340.

10. Westerblad, H., Bruton, J. D. and Katz, A. (2010). Skeletal muscle: energy metabolism, fiber types, fatigue and adaptability. Exp Cell Res 316(18): 3093-3099. 